\title{
Fabrication and thermal stability studies of polyamide 66 containing triaryl phosphine oxide
}

\author{
XIAOFENG YANG, QIAOLING LI*, ZHIPING CHEN and HONGLI HAN \\ Department of Chemistry, School of Science, North University of China, Taiyuan 030051, China
}

MS received 19 July 2008; revised 20 October 2008

\begin{abstract}
An intrinsically halogen-free flame retardant polyamide 66 (FR-PA66) was fabricated successfully by two-step polymerization reaction with adipic acid hexamethylene salt (AH salt) and bis(4-carboxyphenyl) phenyl phosphine oxide (BCPPO) as raw materials. The structure, combustion properties and thermal stability were characterized by means of intrinsic viscosity, Fourier transform infrared spectroscopy (FTIR), combustion testing, differential scanning calorimetry (DSC), thermogravimetric analysis (TG) and scanning electron microscope (SEM). Experiments show that BCPPO have excellent copolymerization properties with AH salt. And incorporation of triaryl phosphine oxide (TPO) did not transform the crystal phase structure of PA66. During fabrication of FR-PA66, melt polymerization time exhibits more surprising influence on intrinsic viscosity than aqueous solution polymerization time. The LOI value of FR-PA66 with 9 wt\% TPO reaches 27.2, and corresponding UL94 rating reaches V-0. Improved thermo-stability of FR-PA66 can be attributed to both forming of compact char protective layer and further consolidation effect of $P / N$ synergistic system.
\end{abstract}

Keywords. Bis(4-carboxyphenyl) phenyl phosphine oxide; halogen-free flame retardant copolyamide 66; fabrication; characterization; thermal stability.

\section{Introduction}

Polyamide 66 (PA66) is an important engineering plastics and has been used for numerous engineering applications due to its favourable properties such as chemical and mechanical resistance, attrition resistance, high tensile and melting point, etc. The flammable of virgin PA66, however, seriously limited the applications of PA66 in some special regions as electron, electric and aviation, which require high temperature, high tension voltage and high load (Sergei and Edward 2000; Zong et al 2003). In order to improve the safety of PA66, numerous research work have been done during last two decades, and most flame retardancy PA66 (FR-PA66) have been utilized (Ilardo and Duffy 1985; Williams 1985, 1987; Theysohn et al 1987; Schmid and Luedi 1990; Bonin and LeBlanc 1991; Van and De 1997) in the market. But most of them were prepared based on flame retardant containing halogen (Ilardo and Duffy 1985; Williams 1985, 1987; Theysohn et al 1987; Bonin and LeBlanc 1991; Van and De 1997). The major problems encountered for this system is the generation of toxic and corrosive fume during combustion (Camino et al 1989; Derout et al 1996). Thus it is desirable for design and fabrication of novel halogen-free anti-flamed PA66.

\footnotetext{
*Author for correspondence (qiaolingl@163.com)
}

Bis(4-carboxyphenyl)phenyl phosphine oxide (BCPPO), which is an high efficiency flame retardant, is a bifunctional compound containing triaryl phosphine oxide. When the monomer, BCPPO, was incorporated chemically into macromolecules, the copolymer exhibits improved flame retardant, hydrolytically stable and thermal stability. The flame resistant PET, prepared via polycondensation reaction of ethylene glocol with BCPPO, exhibits good fibre-forming properties, improved flame retarding behaviour, high glass transition temperatures and improved thermal stability (Wang et al 2000).

In this work, an intrinsically flame retarded co-polyamide 66 were fabricated through the direct amidation and polycondensation reaction of adipic acid hexamethylene salt (AH salt) with 1,6-hexanediamine and BCPPO as co-monomer. The structure, combustion properties and thermal stability of FR-PA66 were investigated.

\section{Experimental}

\subsection{Materials}

The bis(4-carboxyphenyl) phenyl phosphine oxide (BCPPO) was synthesized in our laboratory and was recrystallized using alcohol (95\%) prior to use according to the method of Wang et al (2000). The product was identified by elemental analysis, appropriate spectroscopic analyses and melting point. The elemental analysis was 
performed by VA2IO EL elemental analysis instrument of Elementar co., Germany. The calculated results from the original molecular formula are (\%): C, 65.58; H, 4.13; P, 8.45. The analytical results are (\%): C, 65.67; H, 4.39; $\mathrm{P}$, 8.41. The melting point $\left(T_{\mathrm{m}}\right)$ and the enthalpy of fusion $\left(\Delta H_{\mathrm{f}}\right)$ were determined by differential scanning calorimeter (DSC) on a STA $449 \mathrm{C}$ thermal analysis equipment at a heating rate of $10^{\circ} \mathrm{C} / \mathrm{min}$ in nitrogen. The obtained $T_{\mathrm{m}}$ and $\Delta H_{\mathrm{f}}$ are $337.0^{\circ} \mathrm{C}$ and $16.9 \mathrm{~kJ} / \mathrm{mol}$, respectively (according to Wang et al (2000), the m.p. was $337.4^{\circ} \mathrm{C}$, and the enthalpy of fusion was $17.6 \mathrm{~kJ} / \mathrm{mol}$ ). The purity of BCPPO was analysed by nuclear magnetic resonance (NMR) spectra (DRX300 spectrometer) with DMSO as solvent, the results were: ${ }^{1} \mathrm{H}$ NMR: 7.375-8.080 ( $m$, $13 \mathrm{H}$, 且) $), 13 \cdot 352(w, 2 \mathrm{H},-\mathrm{COOH})$. The proton belonging to methyl groups was not found. Infrared spectrum (IR) was recorded with FTIR-8400S spectrometer using $\mathrm{KBr}$ pellet, the obtained results are: $1245(\mathrm{P}=\mathrm{O}) ; 1716(\mathrm{C}=\mathrm{O})$; $3000(\mathrm{O}-\mathrm{H}) ; 1435$ (P-Ar).

The adipic acid hexamethylene salt (AH salt) (melting point, $196^{\circ} \mathrm{C}$ ) was kindly supplied by Henan HuangLong plastics plant. 1,6-hexanediamine and formylic acid were purchased from reagent company with pure analysis.

\subsection{Fabrication of FR-PA66}

The FR-PA66 was fabricated by a polycondensation reaction of AH salt with BCPPO and 1,6-hexanediamine as co-monomer. The main reaction for fabrication of FRPA66 is expressed in figure 1.

In order to avoid volatilization of 1,6-hexanediamine and improve polymerization degree, two-stage polycondensation reaction was performed. At the start of the reaction, aqueous solution polymerization was performed as following procedure. The weighted $\mathrm{AH}$ salt, deionized water and certain quality BCPPO relative to $\mathrm{AH}$ salt were blended, and the $\mathrm{pH}$ value was adjusted to 7.5 with $1,6-$ hexanediamine. Then the mixtures were charged into polymerization autoclave (Weihai-controlled reactor Ltd, WHF-2) equipped with mechanical stirring, a gas inlet

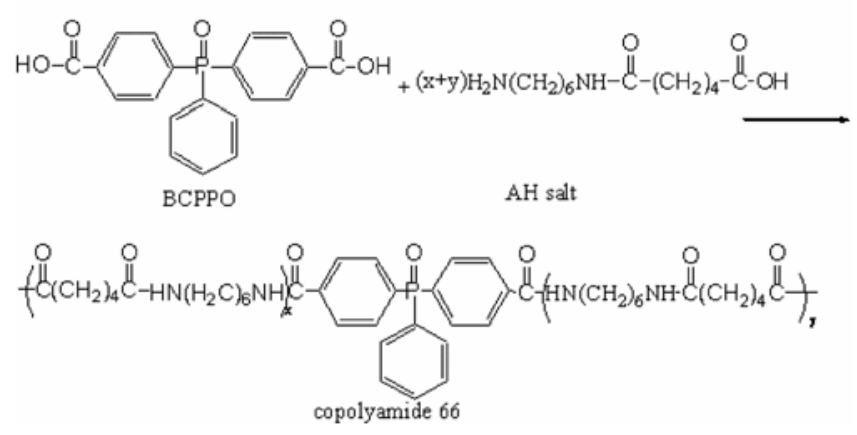

Figure 1. Fabrication process of FR-PA66. and a gas outlet. The atmospheric oxygen present inside the autoclave was removed by repeated rinsing under pressure with purified nitrogen. Next autoclave was heated to $230^{\circ} \mathrm{C}$ and stirred with $50 \mathrm{r} / \mathrm{min}$ for $90 \mathrm{~min}$ under $1.75 \mathrm{MPa}$ pressure. With proceeding of the polymerization reaction, the volatilized component decreased, and the oligopolymer increased gradually. And melt polymerization was employed to complement the second stage of polycondensation reaction. In this stage, the reactor is decompressed firstly to ambient pressure meanwhile heated to $275^{\circ} \mathrm{C}$. Then the polymerization was kept under vacuum and stirring conditions for $25 \mathrm{~min}$ at $275^{\circ} \mathrm{C}$. After melt polymerization, the molten copolymer was drawn through the bottom valve under certain pressure, cooled and granulated.

\subsection{Characterization of FR-PA66}

2.3a Intrinsic viscosity: The molecular masses of the FR-PA66 are expressed by their intrinsic viscosities, which were determined from measurements made on solutions of the copolymers $(c=0.5,0.33,0.25,0.17$ and $0.12 \mathrm{~g} / 100 \mathrm{ml}$ in formylic acid) with Ubbelodhe viscometer at $25^{\circ} \mathrm{C}$.

To optimize copolymerization technology, copolymerization time in different stages and TPO content were changed, respectively. And intrinsic viscosity was determined accordingly to above measurements.

2.3b FT-IR: In order to eliminate the influence of unreacted BCPPO co-monomer, FR-PA66 was first handled with file and power was obtained. Then power was extracted with sodium hydroxide solution and deionized water, respectively. Then the structure of FR-PA66 was characterized on $\mathrm{KBr}$ pellets using FTIR-8400S spectrometer.

2.3c Combustion test: The vertical burning test was conducted on a CZF-3 horizontal and vertical burning tester, on sheets $127 \times 12.7 \times 3.2 \mathrm{~mm}$ and $127 \times 12.7 \times$ $1.6 \mathrm{~mm}$ according to the America National UL 94 test, ASTM D3801. The LOI value was obtained using an ATLAS limiting oxygen index instrument on sheets $120 \times 6.5 \times 3.2 \mathrm{~mm}$ according to the standard oxygen index test, ASTM D2863-70.

2.3d DSC-TG: The melting point and thermal mass losing behaviour of FR-PA66 were characterized by employing a Netzsch differential scanning calorimeter (Model 449C) with a heating rate of $10^{\circ} \mathrm{C} / \mathrm{min}$ from room temperature to $600^{\circ} \mathrm{C}$. And the measurements were performed under a nitrogen atmosphere.

2.3e Analysis of char residue surface: Char residue of FR-PA66 were obtained by ignition samples on $600^{\circ} \mathrm{C}$ 
for $2 \mathrm{~h}$ in muffle furnace. SEM was used to characterize the char surface of the FR-PA66. SEM photographs were taken on a Hitachi S-530 SEM with an accelerating voltage of $25 \mathrm{kV}$.

\section{Results and discussion}

\subsection{Process of FR-PA66}

3.1a Effect of reaction time on intrinsic viscosity: The effect of copolymerization time on intrinsic viscosity at different stages is shown in figure 2. Where the intrinsic viscosity with square is obtained by fixed melt polymerization time for $25 \mathrm{~min}$, and correspondingly the intrinsic viscosity with circle is obtained by fixed aqueous solution polymerization time for $90 \mathrm{~min}$. From figure 2, we can find that copolymerization time has same influence on intrinsic viscosity in different stages. With extension of polymerization time, the intrinsic viscosity of FRPA66 increases gradually. Compared to the variety of reaction time in aqueous solution polymerization, however, the variety of polymerization time in melt polymerization exhibits greater influence on intrinsic viscosity. This phenomenon is mainly caused by the difference of reaction temperature during two stages. At the early stage of polymerization, the temperature of system was lower, and the reaction was single. So it is easy relatively for controlling the structure and molecular weight of copolymer. As polymerization proceeded, the temperature was raised at the second stage. Subsidiary reactions such as crosslinking and decarboxylation increase with prolonging polymerization time (Pan 2003). In general, the intrinsic viscosity of PA66 in formylic acid was preferably in the range of $1.2-1.6 \mathrm{dL} \cdot \mathrm{g}^{-1}$ when the PA66 was used as engineering plastic. From this view point, appropriate copolymerization time for preparation of FR-

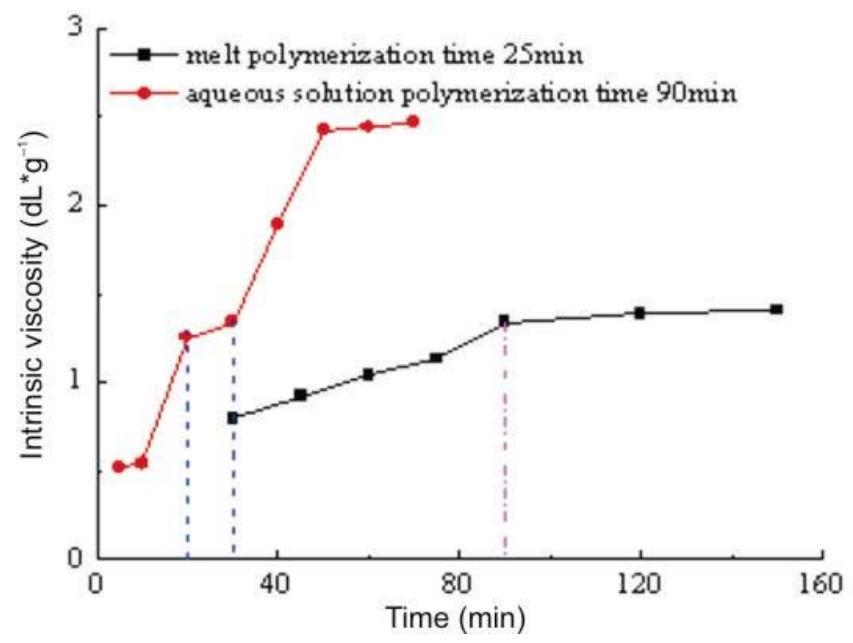

Figure 2. Variation of the intrinsic viscosity with copolymerization time.
PA66 is $90 \mathrm{~min}$ in aqueous solution polymerization stage and $25 \mathrm{~min}$ in melt polymerization stage, respectively according to our experiments.

3.1b Effect of TPO content on intrinsic viscosity: The effect of TPO content on intrinsic viscosity is shown in figure 3 . Here aqueous solution polymerization time and melt polymerization time were fixed as $90 \mathrm{~min}$ and $25 \mathrm{~min}$, respectively. It can be seen from figure 3 that the intrinsic viscosity of FR-PA66 increased smoothly with addition of TPO content under same polymerization condition. This was caused by the difference of competitive rate of raw materials. According to the theory of isoactivity for function, the function activity has nothing to do with length of the segment (Pan 2003). So the activity of function is mainly decided by the groups connected directly with function. Compared to $\mathrm{AH}$ salts, the carboxyl of BCPPO connected with aromatic group. Due to conjugated effect of aromatic group, nuclophilic reactivity of BCPPO is higher than $\mathrm{AH}$ salt. Therefore, the copolymerization rate became greater with the incorporation of BCPPO at the same polymerization condition, and resulted in higher molecular masses of FR-PA66, which were reflected by higher intrinsic viscosity. That is to say, BCPPO have excellent copolymerization properties with $\mathrm{AH}$ salt.

\subsection{Structure analysis of FR-PA66}

A typical DSC thermogram of FR-PA66 and PA66 is shown in figure 4 . As can be seen, there is only a single endothermic peak for FR-PA66 before thermolysis, which is assigned to the melting temperature $\left(T_{\mathrm{m}}\right)$. This phenomenon indicated that the copolymerization of $\mathrm{AH}$ salt with BCPPO formed just a random copolymer but not a homopolymer of PA66. Observe the thermogram carefully, it can also be found that the $T_{\mathrm{m}}$ of FR-PA66 $\left(258^{\circ} \mathrm{C}\right)$ is lower than that of homopolymer of PA66

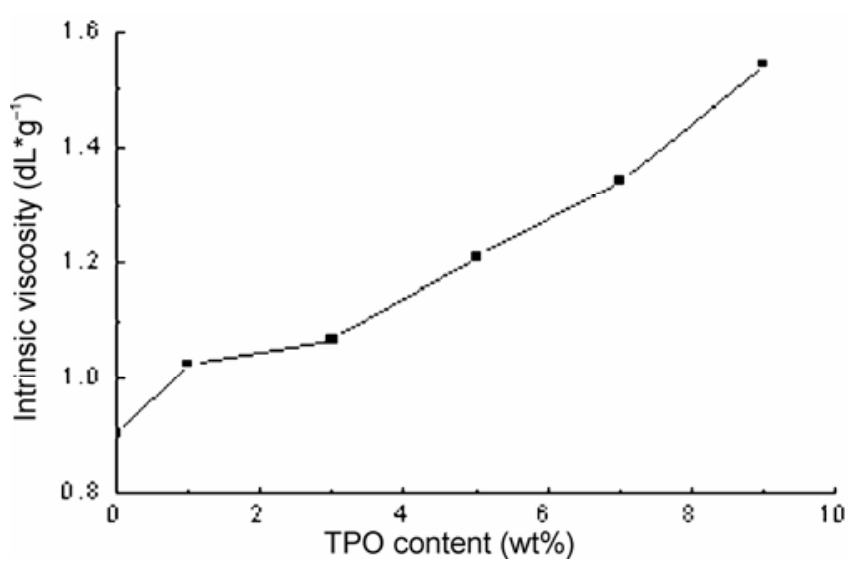

Figure 3. Effect of $\mathrm{BCPPO}$ content on intrinsic viscosities of FR-PA66. 
$\left(268^{\circ} \mathrm{C}\right)$. These findings can be understood on the change of conformation of PA66. Since in the $\alpha$ form of PA66 the molecular chains were of the fully extended planar zigzag, the intermolecular phase angle can assume values of zero or $180^{\circ}$, which results to form hydrogen bonding more easily. As the monomer of BCPPO was incorporated into PA66 chains, it deviates the intermolecular phase angle of PA66, which resulted in the progressive attenuation of the intermolecular interaction (hydrogen bonding) between PA66 molecules and thus caused a lowering of $T_{\mathrm{m}}$.

Typical FT-IR spectra of PA66 and FR-PA66 were shown in figure 5 . It showed the typical polyamide peaks at: $3301 \mathrm{~cm}^{-1}\left(\mathrm{~N}-\mathrm{H}\right.$ stretching), $1635 \mathrm{~cm}^{-1}$ (C=O stretching, amide I), $1541 \mathrm{~cm}^{-1}(\mathrm{~N}-\mathrm{H}$ bending, amide II) and $1473 \mathrm{~cm}^{-1}\left(\mathrm{CH}_{2}\right.$ bending). In addition, peaks appearing at 686 and $578 \mathrm{~cm}^{-1}$, which were assigned to amide $\mathrm{V}$ and amide VI in $\alpha / \beta$ phase, respectively indicated that FRPA66 maintains the crystal structure of PA66. Further

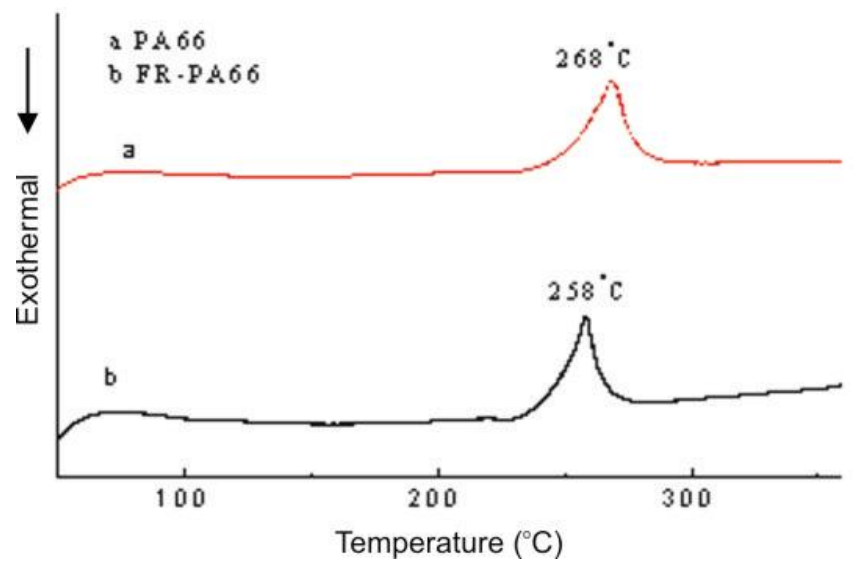

Figure 4. Typical DSC curves of PA66 and FR-PA66.

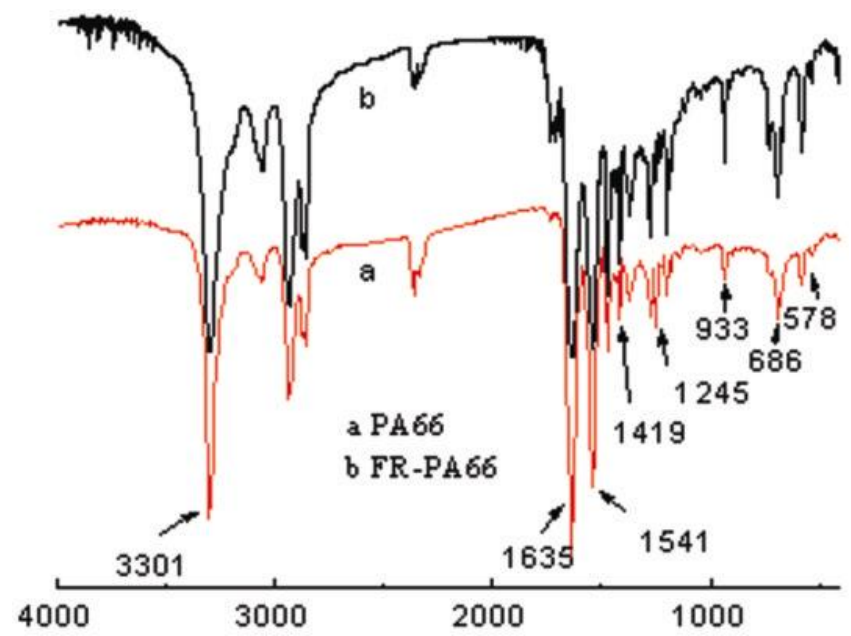

Figure 5. FT-IR spectra of PA66 and FR-PA66. proof can also be found at about 1541,1419 and $933 \mathrm{~cm}^{-1}$, which is attributed to the $\alpha$ phase of PA66 according to the literature (Wang et al 2007). Therefore, the incorporation of BCPPO might perturb the intermolecular interaction of hydrogen bonding between polyamides molecules, but not transform the crystal phase structure of PA66. By contrasting the spectra of PA66 and FR-PA66 carefully, a sharp peak at $1245 \mathrm{~cm}^{-1}(\mathrm{P}=\mathrm{O}$ stretching) was found in FR-PA66. The fact indicated that there was constitutional unit of BCPPO in main chain of copolymer. And BCPPO has been incorporated into PA66 successfully by copolymerization reaction.

\subsection{Flame retardancy and thermal stability of FR-PA66}

Table 1 shows the combustion test results of both PA66 and FR-PA66. When TPO content is $3 \mathrm{wt} \%$, the LOI value of FR-PA66 can reach $24 \cdot 5$, two units higher than that without TPO, meanwhile, the corresponding UL94 rating is improved from $\mathrm{V}-2$ to part $\mathrm{V}-0$. With increase of TPO content from 3 to $9 \mathrm{wt} \%$, the LOI value of PA66 is enhanced from 24.5 to 27.2 by 2.7 units, corresponding UL94 rating reached V-0. FR-PA66 with $9 \mathrm{wt} \%$ TPO exhibits nearly similar combustion properties to PA66 with $10 \mathrm{wt} \%$ MERP (Liu and Wang 2006) (80 wt\% red phosphorus and $20 \%$ melamine cyanurate). Above result indicates that FR-PA66 has excellent flame retardancy.

Figure 6 and table 2 are the results of weight loss and thermal degradation with respect to PA66 and FR-PA66, respectively. Where $T_{10 \%}, T_{50 \%}$ and $T_{90 \%}$ denote the temperature at weight loss $10 \%, 50 \%$ and $90 \%$, respectively and $T_{\max }^{1}$ and $T_{\max }^{2}$ denote the temperature at the first and second maximum weight loss, respectively. From table 2 , we found that the thermolysis temperature of FR-PA66 is approximately $10^{\circ} \mathrm{C}$ higher than virgin PA66 at same weight loss, as well as the mass residue is high by about 5\%. Therefore, FR-PA66 exhibits improved thermostability than virgin PA66 significantly. By contrasting

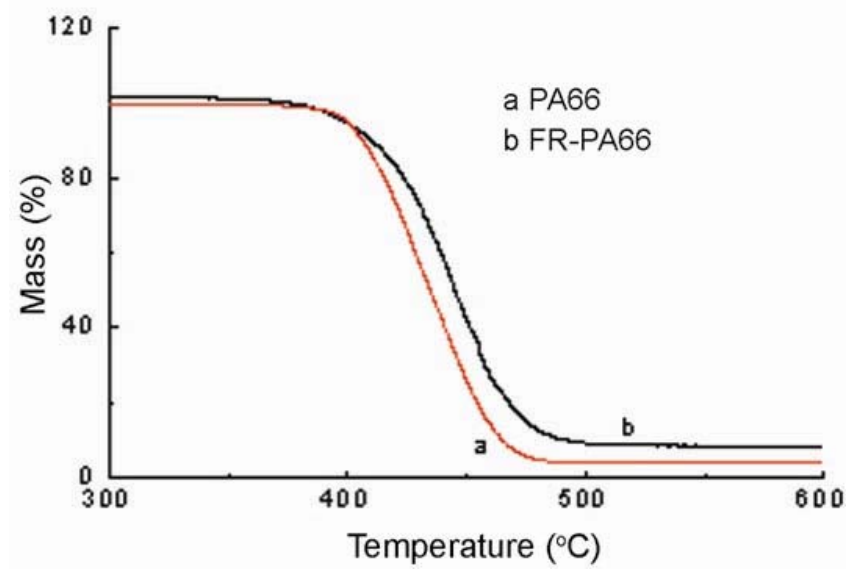

Figure 6. Typical TG curves of PA66 and FR-PA66. 
Table 1. Combustion properties of FR-PA66.

\begin{tabular}{|c|c|c|c|c|c|}
\hline Materials & & Virgin PA66 & $\begin{array}{c}\text { PA66 } \\
\text { (3 wt\% TPO) }\end{array}$ & $\begin{array}{c}\text { PA66 } \\
\text { (7 wt } \% \text { TPO) }\end{array}$ & $\begin{array}{c}\text { PA66 } \\
\left(10 \text { wt } \% \text { MERP }^{b}\right)\end{array}$ \\
\hline LOI & & $22 \cdot 3$ & $24 \cdot 5$ & $27 \cdot 2$ & $27 \cdot 5$ \\
\hline \multirow[t]{2}{*}{ UL94 at $3.2 \mathrm{~mm}$ thickness } & $\mathrm{tf}^{\mathrm{a}}(\mathrm{s})$ & - & - & $10 \cdot 3$ & - \\
\hline & rating & $\mathrm{V}-2$ & Part V-0 & $\mathrm{V}-0$ & - \\
\hline \multirow[t]{2}{*}{ UL94 at $1.6 \mathrm{~mm}$ thickness } & $\mathrm{tf}^{\mathrm{a}}(\mathrm{s})$ & $88 \cdot 0$ & - & $26 \cdot 7$ & $28 \cdot 2$ \\
\hline & rating & $\mathrm{V}-2$ & Part V-0 & V-0 & $\mathrm{V}-0$ \\
\hline
\end{tabular}

${ }^{a}$ Means the total duration (five specimen) of flaming combustion; ${ }^{b}$ MERP was prepared with $80 \mathrm{wt} \% \mathrm{RP}$ (red phosphorus) and $20 \mathrm{wt} \% \mathrm{MCA}$ (melamine cyanurate) as raw materials.

Table 2. Comparison of thermal degradation properties of PA66 and FR-PA66.

\begin{tabular}{lcccccc}
\hline Sample & $T_{10 \%}\left({ }^{\circ} \mathrm{C}\right)$ & $T_{50 \%}\left({ }^{\circ} \mathrm{C}\right)$ & $T_{90 \%}\left({ }^{\circ} \mathrm{C}\right)$ & $T_{\max }^{1}\left({ }^{\circ} \mathrm{C}\right)$ & $T_{\max }^{2}\left({ }^{\circ} \mathrm{C}\right)$ & $\mathrm{CR}\left(550^{\circ} \mathrm{C}\right)\left({ }^{\circ} \mathrm{C}\right)$ \\
\hline PA66 & $406 \cdot 6$ & 434.6 & $465 \cdot 1$ & 427.6 & $440 \cdot 5$ & 3.92 \\
FR-PA66 & $410 \cdot 2$ & $445 \cdot 1$ & 491.9 & 443.0 & 455.9 & 8.94 \\
\hline
\end{tabular}

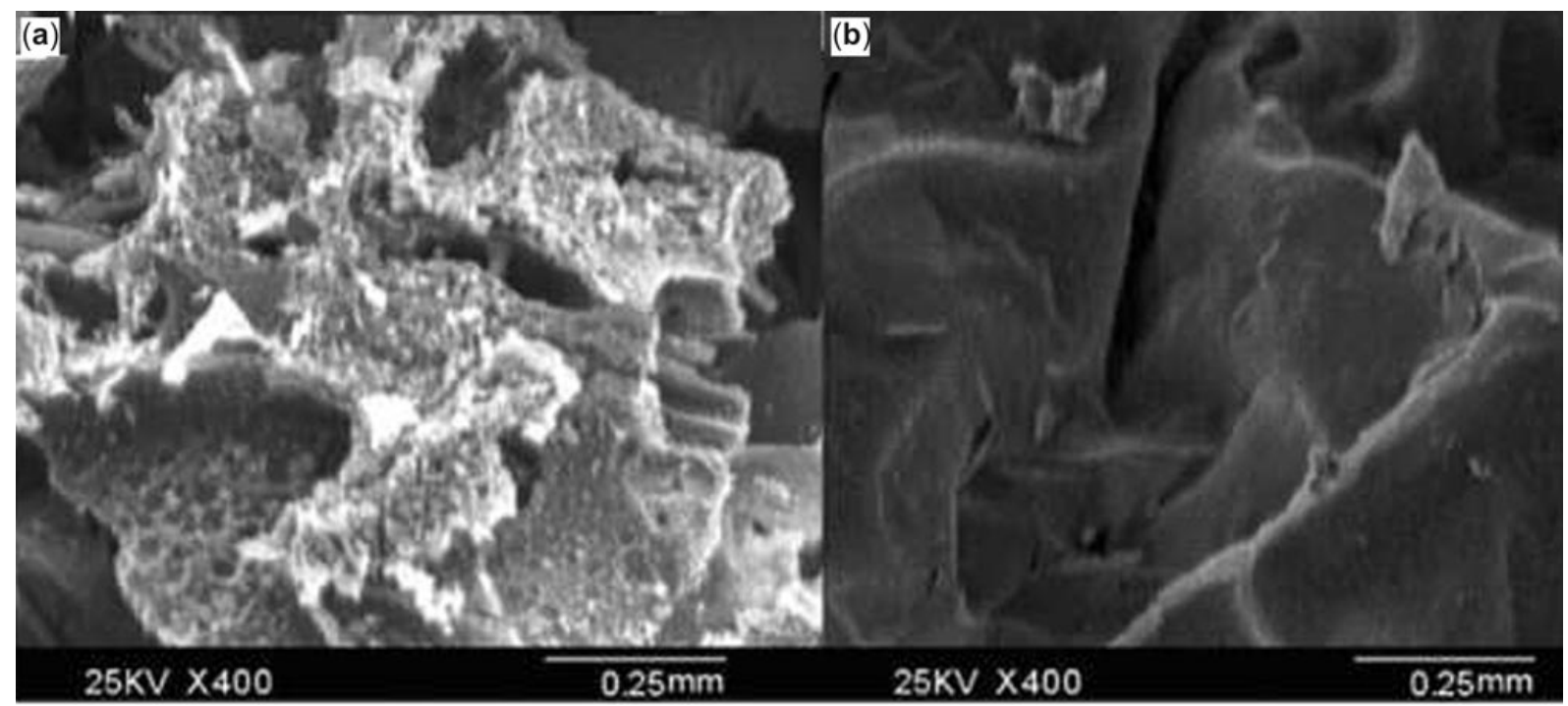

Figure 7. SEM of char residues of (a) PA66 and (b) FR-PA66.

the SEM photograph of FR-PA66 and PA66 in figure 7, we can also find that the char surface of FR-PA66 is ceramic-like, inorganic-rich, compact, refractory and inflammable, while the char surface of PA66 is loose and accompanying with many large apertures.

We think that the improved thermo-stability of FRPA66 can be explained as follows: Firstly, when FRPA66 exposed to flaming temperature in nitrogen, organophorus polyamide thermolysis, and formed super dehydration property of polyphospharic acid. On the one hand, polyphospharic acid having unevaporable heavygravity properties covers the surface of coacervated phase, and interrupted the fuel, oxygen and heat transfer (Kuo et al 1998; Horold 2000). On the other hand, polyphospharic acid reacted with polyamide and/or its degra- tion product to yield a compact char protective layer (Horold 2000). Secondly, due to synergistic effect of $\mathrm{P} / \mathrm{N}$, organic phosphorus reacted with nitrogen-containing compound derived from PA66, and formed macromolecular substances such as $(\mathrm{PNO})_{x}$ or $(\mathrm{PN})_{x}$ with high thermal stability (Costa and Camino 1990). These macromolecular substances further consolidated the char protective layer. Finally, higher density aromatic ring of TPO also render improvement of mass residue of FR-PA66.

\section{Conclusions}

In summary it can be drawn that the present flame retardant BCPPO has an excellent copolymerization property with $\mathrm{AH}$ salt in the fabrication of FR-PA66. At the same 
time, incorporation of BCPPO might perturb the intermolecular interaction (hydrogen bonding) between PA66 molecules, but not transform the crystal phase of PA66. The intrinsic viscosity of FR-PA66 was influenced by TPO content, aqueous solution copolymerization time and melt copolymerization time. But melt polymerization time exhibits greater influence on intrinsic viscosity. With introduction of TPO, FR-PA66 exhibits improved thermo-stability. The LOI value of FR-PA66 with 9wt\% TPO reaches $27 \cdot 2$, and corresponding UL94 rating reaches V-0. When FR-PA66 is exposed to flaming temperature, a char protective layer is formed on the surface of material and further consolidated by $\mathrm{P} / \mathrm{N}$ synergistic effect. The protective layer interrupted the fuel, oxygen and heat transfer, and improved the thermolysis temperature and mass residue of PA66.

\section{Acknowledgements}

The work has been supported by Program for the Top Science and Technology Innovation Teams of Higher Learning Institutions of Shanxi and Foundation of North University of China.

\section{References}

Bonin Y and LeBlanc J 1991 US Patent 4985485.01151991 Camino G, Costa L and Martinasso G 1989 Polym. Degrad. Stab. 23359

Costa L and Camino G 1990 Fire and polymers, in ASC symposium series (Washington DC) Vol. 425, pp 211-238

Derout D, Morvan F and Bross J C 1996 J. Appl. Polym. Sci. 62 1855

Horold S 2000 Polym. Degrad. Stab. 36427

Ilardo C S and Duffy J J 1985 US Patent 4504611.03121985

Kuo P L, Chang J M and Wang T L J 1998 J. Appl. Polym. Sci. 691635

Liu Yuan and Wang Qi 2006 Polym. Degrad. Stab. 913103

Pan Z R 2003 Polymer chemistry (Chemical Industry Press) 3rd edn., pp. 195, 197

Schmid E and Luedi D 1990 US Patent 4963610.10161990

Sergei V L and Edward D W 2000 Polym. Int. 491033

Theysohn R et al 1987 US Patent 4076682.02281987

Van W L and De S D 1997 US Patent 5674972.10071997

Wang L, Wang X and Yan G 2000 Polym. Degrad. Stab. 69127

Wang X H, Fang X L, Yao C and Wu F C 2007 Mod. Chem. Ind. 2736

Williams I G 1985 US Patent 4552912.11121985

Williams I G 1987 US Patent 4696966.09291987

Zong R W, Hu Y and Wang S F 2003 Fire Safety Science 1246 